\title{
Valoración de los estudiantes sobre su formación en la Facultad de Medicina
}

\author{
José A. Mirón-Canelo, Helena Iglesias-De Sena, Montserrat Alonso-Sardón
}

Objetivo. Conocer y analizar la valoración y las opiniones que tienen los alumnos de sexto curso de medicina sobre el proceso de enseñanza-aprendizaje en su facultad.

Sujetos y métodos. Se realiza un estudio transversal a partir de los datos primarios aportados por una encuesta que se aplica a 225 alumnos de sexto de medicina.

Resultados. Del total de encuestados, 208 alumnos (95\%; IC 95\% = $95 \pm 3$ ) no se consideran formados para ejercer la medicina y $214(96 \%$; IC 95\% = $96 \pm 3)$ no se encuentran capacitados para ejercerla. La calidad global de la formación recibida en el pregrado es valorada por el 55\% (IC 95\% = $55 \pm 7$ ) de los alumnos con una puntuación media de 3 en una escala de 1 a 5 . Se valora positivamente la formación teórica y negativamente las habilidades clínicas, sociales y de comunicación, las actitudes y los aspectos relacionados con la gestión. Para 169 alumnos, un $75 \%$ (IC 95\% = $75 \pm 6$ ), la prueba MIR ha condicionado la formación, tanto en contenidos (70\%; IC 95\% = $70 \pm 3$ ) como en metodología (58\%; IC 95\% = $58 \pm 6$ ).

Conclusiones. Globalmente, existe una evolución positiva entre 1999 y 2008-2010. Los resultados de este trabajo ponen de manifiesto la necesidad de introducir cambios en el proceso de enseñanza-aprendizaje de la medicina que mejoren la formación efectiva y la satisfacción.

Palabras clave. Educación médica. Estudiantes. Formación universitaria.

\section{Valuation of the students about his formation in the Faculty of Medicine}

Aim. To know and to analyze the knowledge and opinions that have the pupils of sixth year of medicine on the educationlearning of the master.

Subjects and methods. A transversal study is realized from the primary information contributed by a survey that is applied to 225 pupils of sixth degree of Medicine.

Results. Of the whole of polled pupils, 208 ( $95 \% ; 95 \% \mathrm{Cl}=95 \pm 3$ ) is not considered to be formed to exercise the Medicine, and 214 (96\%; 95\% Cl = $96 \pm 3$ ) is not qualified to exercise the Medicine. The global quality of the formation received in the pregrade is valued by $55 \%(95 \% \mathrm{Cl}=55 \pm 7)$ of the pupils by an average score of 3 for a scale of $1-5$. With regard to the formative components, the theoretical formation is valued very positively and negatively the clinical and social skills and the communication, the attitudes and the aspects related to the management. The MIR test has determined as methodology. For 169 pupils ( $75 \% ; 95 \% \mathrm{Cl}=75 \pm 6)$, the MIR test has determined formation so much contained (70\%; $95 \% \mathrm{Cl}=70 \pm 3)$ as methodology $(58 \% ; 95 \% \mathrm{Cl}=58 \pm 6)$.

Conclusions. Globally, a positive evolution exists between 1999 and 2008-2010. The results of this work reveal the need to introduce changes in the process of education learning of the Medicine that the effective formation and satisfaction improve.

Key words. Medical education. Students. University education.

\section{Introducción}

En la actualidad, la salud se encuentra entre las mayores preocupaciones de la población, por lo que forma parte de los objetivos prioritarios del estado del bienestar y por ello, las facultades de medicina deben conseguir profesionales sanitarios que intervengan de forma adecuada como médicos, en el conjunto del sistema sanitario español. La educación médica se convierte así en una cuestión de gran interés e impacto social, que tiene como objetivo desarrollar la competencia profesional y las ac-
Departamento de Medicina Preventiva, Salud Pública y Microbiología Médica. Facultad de Medicina. Universidad de Salamanca. Salamanca, España.

Correspondencia: Dr. José Antonio Mirón Canelo. Departamento de Medicina Preventiva, Salud Pública y Microbiología Médica. Facultad de Medicina. Universidad de Salamanca. Alfonso X el Sabio, s/n. E-37007 Salamanca.

E-mail: miroxx@usal.es (c) 2011 Educación Médica 
Tabla I. Cuestionario.

1. Sexo: Varón/Mujer

2. Edad (años)

3. ¿Se considera suficientemente formado como médico? Sí/No. ¿Por qué?

4. ¿Se considera formado y capacitado para ejercer la medicina? Sí/No. ¿Por qué?

5. Valore los distintos elementos que intervienen en la relación clínica a

Trato humano

Competencia y habilidades clínicas

Habilidades sociales y de comunicación

Equipamiento e instalaciones

Interés por curar y tratar adecuadamente

Otros

6. Valore los distintos componentes de la formación pregrado de nuestra facultad ${ }^{a}$

Conocimientos teóricos

Habilidades clínicas y técnicas

Habilidades sociales y de comunicación

Actitudes profesionales

Aspectos éticos

Otros

7. Valore los distintos componentes de su formación pregrado a

Conocimientos teóricos

Habilidades clínicas y técnicas

Habilidades sociales y de comunicación

Actitudes profesionales

Aspectos éticos

Otros

8. ¿Le ha preocupado o se ha interesado en algún momento de su formación y preparación por alguno de estos componentes de su formación que considera importante? Sí/No. ¿Por cuál?

9. ¿En los estudios de licenciatura se favorece el autoaprendizaje? Sí/No

10. ¿Le parece que la formación pregrado está condicionada por el examen MIR? Sí/No. ¿Desde qué curso? ¿En qué grado? ${ }^{a}$

11. ¿Le parece que los contenidos del pregrado están condicionados por el examen MIR? Sí/No. ¿Desde qué curso? ¿En qué grado? a

12. ¿Le parece que los métodos y técnicas de la enseñanza de pregrado están condicionados por el examen MIR? Sí/No. ¿Desde qué curso? ¿En qué grado? a

13. ¿Su aprendizaje ha estado condicionado por el examen MIR? Sí/No. ¿Desde qué curso?

14. ¿Le parece el examen MIR un adecuado sistema de selección para la formación especializada o posgrado? Sí/No

15. ¿Le parece que en el examen MIR deberían introducirse cambios? Sí/No

16. ¿Le parece que existe colaboración y coordinación entre las áreas de conocimiento (asignaturas) del pregrado? Sí/No

17. ¿Le parecen adecuados los sistemas de evaluación del pregrado? Sí/No

18. ¿Le parece que los materiales y equipos utilizados en la formación del pregrado son adecuados? Sí/No

19. ¿Qué material o fuente de conocimientos utiliza en su formación en las distintas áreas del conocimiento? a

Libros

Revistas

Apuntes elaborados

Apuntes de clase

Base de datos

Internet

Otros

20. Valore la calidad de la formación pregrado global ${ }^{a}$

21. Valore la calidad global de la formación pregrado preclínica (1.․ 2 2.ำ y $3 . \circ$ a a

titudes que favorecen la mejor atención sanitaria de la población $[1,2]$. Parece bastante obvia la interconexión entre la educación médica, la formación de los profesionales, la asistencia sanitaria y la salud de la población; lo que no está tan claro es hasta dónde puede llegar cada uno de ellos.

La formación de los profesionales sanitarios está considerada como un continuo que se extiende desde el bachillerato hasta que se abandona el ejercicio de la profesión. Ésta se divide en tres etapas fundamentales (grado o licenciatura; posgrado y especialidad; formación continua y desarrollo profesional continuo), que constituyen la cadena educativa médica. La primera etapa tiene lugar en las facultades, donde se da una formación básica e integral en los fundamentos de la medicina; se ponen los pilares básicos y la estructura troncal del futuro profesional. Por ello es necesario reflexionar, ser flexibles y adaptarse a los requerimientos específicos de cada momento y situación docente [3].

Desde que se creó la primera universidad en Salerno, en el año 1098, hasta nuestros días han pasado muchas cosas y han cambiado notablemente los contenidos profesionales y los medios destinados a la enseñanza de la medicina.

Aceptando la idea básica de que los estudios de medicina deben buscar la capacitación para el ejercicio profesional correcto y adecuado, la primera etapa, de formación del licenciado o grado, se considera el período formativo más fértil e imprescindible en la adquisición de los fundamentos básicos y sólidos de la medicina [4]. Por ello, esta etapa es crucial en la formación de todo médico y es, a su vez, la gran asignatura pendiente [2].

Los nuevos planes de estudio que se han establecido en las universidades españolas por el proceso de adaptación al Espacio Europeo de Enseñanza Superior (EEES) proponen una potenciación de la formación del grado, al objeto de formar médicos capaces de tomar decisiones y dar consejos sobre los problemas de salud de la población [5]. Se cambia el proceso de enseñanza-aprendizaje para incentivar la adquisición de competencias y promocionar el autoaprendizaje.

El objetivo de este trabajo ha sido conocer la valoración que hacen los estudiantes del grado de medicina, para lo cual se analizan y se valoran las opiniones que dan los alumnos.

\section{Sujetos y métodos}

Se realiza un estudio transversal, a través de un cuestionario autocumplimentado [6]. La población sometida a estudio fueron los alumnos de sexto, de dos cursos académicos consecutivos, de la licenciatura de Medicina y Cirugía de la Facultad de Medicina de Salamanca (curso académico 2008-2009 y 2009-2010). 
La encuesta fue cumplimentada por 225 alumnos que cursaban el último curso de licenciatura, según los contenidos establecidos en el plan de estudios de 2001 [7]. Representan el 70\% del total de alumnos matriculados durante estos dos cursos. La participación fue voluntaria y consistió en cumplimentar un cuestionario estructurado de 30 ítems, la mayoría de ellos cerrados. El cuestionario utilizado en este trabajo hace referencia a varias áreas de investigación sobre su formación universitaria: aspectos generales (ítems 3, 4, 5, 8, 9), formación y prueba MIR (ítems 10-15), formación y facultad de medicina (ítems 1619,26 y 27) y valoraciones (ítems 6, 7, 20-25). Contiene posibles respuestas en una escala numérica cuantitativa con opciones entre un máximo de 5 y un mínimo de 1 (Tabla I). Este trabajo fue validado en un estudio previo realizado en 1999 [8,9].

La encuesta se aplicó un día de clase al azar, a los alumnos que estaban en el aula, dentro del último mes lectivo, y el tiempo medio que emplearon los alumnos en cumplimentarla fue de 11 minutos.

Los datos se introdujeron en el programa estadístico SPSS v. 16.0 para facilitar su procesamiento y análisis. Posteriormente, se clasificaron y tabularon para facilitar su valoración e interpretación. Las frecuencias se presentan en porcentajes, con sus intervalos de confianza del 95\% (IC 95\%).

\section{Resultados}

El total de alumnos encuestados fue de 225, de los cuales 70 (31\%) fueron varones y 155 (69\%) mujeres. La edad media fue de 23,41 $\pm 1,125$ años.

Las valoraciones generales sobre la calidad de la enseñanza universitaria recibida se presentan en la tabla II. La calidad global de la formación del pregrado se valora con una puntuación 3 por el 55\% (IC $95 \%=55 \pm 7$ ) de los alumnos. El nivel de satisfacción por la formación recibida es superior a 3 para el $10,4 \%$ (IC 95\% = 10,4 \pm 4 ). Respecto a los componentes, se valora de forma positiva solamente la formación teórica ( $53 \%$; IC 95\% = $53 \pm 7$ ) y los aspectos éticos (51\%; IC 95\% = $51 \pm 7$ ), y de forma negativa, las habilidades clínicas y técnicas (71\%; IC 95\% = $71 \pm$ 6) y las actitudes profesionales ( $42 \%$; IC $95 \%=42 \pm 6$ ).

La tabla III presenta los aspectos que, en opinión de los estudiantes, deben mejorarse. Destacan las relaciones entre profesores y alumnos, la gestión y planificación, y los métodos de enseñanza. Respecto a las fuentes de conocimiento que han utilizado como apoyo en su formación o enseñanza, destaca el hecho de que la mayoría recurren básicamente a apuntes de clase $(93 \%$; IC $95 \%=93 \pm 3)$ y a libros $(47 \%$;
Tabla I. Cuestionario (cont.).

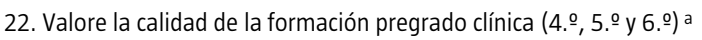

23. Valore los aspectos de gestión, planificación y administración de la formación pregrado a

24. Valore los factores que deben ser mejorables ${ }^{a}$

Métodos

Relaciones alumnos-profesores

Recursos

Gestión y planificación académica

Sistemas de evaluación

Otros

25. Valore su satisfacción personal con la formación pregrado

26. ¿Conoce los cambios que se van a introducir en relación con la adaptación al Espacio Europeo de Educación Superior? Sí/No. ¿Cuáles?

27. ¿Qué cambios deberían introducirse, en su opinión, en los planes de estudio del pregrado?

28. Media del expediente aproximado (MH: 4; S: 3; N: 2; A: 1)

29. ¿Qué especialidad quiere realizar si sus resultados en el examen MIR se lo permiten?

Médica. ¿Cuál?

Quirúrgica. ¿Cuál?

Atención primaria. ¿Cuál?

Otras. ¿Cuál?

30. ¿Tiene previsto realizar el doctorado? Sí/No. ¿Por qué? ¿Cuál?

31. Señale todo aquello que considere necesario para mejorar la calidad de los estudios del pregrado

a En una escala de 1 (peor valoración) a 5 (mejor valoración).

IC 95\% = $47 \pm 7$ ), siendo mínimo el número de alumnos que utiliza revistas y maneja bases de datos.

En la tabla IV se reflejan las valoraciones y opiniones sobre las influencias de la prueba selectiva de residentes y los estudios de pregrado. La mayoría, 169 alumnos (IC 95\% = $75 \pm 6$ ), piensan que la prueba MIR condiciona el desarrollo de los estudios del pregrado. En opinión de 185 alumnos (IC 95\% = 84 \pm 5 ) deberían introducirse cambios en los estudios del pregrado y tan sólo 34 alumnos $(15,5 \%)$ dicen conocer los cambios que se van a introducir en relación con la adaptación al EEES. Al 51\% (IC 95\% = 51 \pm 7 ) le parecen adecuados los sistemas de evaluación; al 28,4\% (IC 95\% = 28,4 \pm 6 ), los materiales y equipos utilizados, y al 9,3\% (IC 95\% = 9,3 \pm 4 ), la colaboración y coordinación entre departamentos.

La tabla V presenta los resultados obtenidos en el año 1999 y 2008-2010. En cuanto a si les parece que los contenidos del pregrado están condicionados por el examen MIR en los años 2008-2010, el 70\% (IC $95 \%=70 \pm 6$ ) respondieron afirmativamente, mientras que en el año 1999 este porcentaje era ligeramente mayor $(73 \%$; IC 95\% = $73 \pm 8)$. Un $75 \%$ de los encuestados manifiesta que el pregrado está condicionado por el examen MIR, mientras que en 1999 este porcentaje era del $87 \%$. Ambos estudios coinciden en la introducción de cambios en esta prueba. 
Tabla II. Aspectos generales del pregrado.

\begin{tabular}{|c|c|c|c|c|c|}
\hline \multirow{3}{*}{ Ítems } & \multicolumn{5}{|c|}{ Valoración general } \\
\hline & 1 & 2 & 3 & 4 & 5 \\
\hline & $n(\% \pm \mathrm{IC} 95 \%)$ & $n(\% \pm \mathrm{IC} 95 \%)$ & $n(\% \pm \mathrm{IC} 95 \%)$ & $n(\% \pm I C 95 \%)$ & $n(\% \pm \mathrm{IC} 95 \%)$ \\
\hline 20. Calidad de la formación pregrado & $11(5 \pm 3)$ & $68(31 \pm 6)$ & $121(55 \pm 7)$ & $21(10 \pm 4)$ & \\
\hline 21. Calidad de la formación pregrado preclínica & $21(9 \pm 4)$ & $62(28 \pm 6)$ & $79(35 \pm 6)$ & $56(25 \pm 6)$ & $6(3 \pm 2)$ \\
\hline 22. Calidad de la formación pregrado clínica & $35(16 \pm 5)$ & $59(27 \pm 6)$ & $83(37 \pm 6)$ & $44(20 \pm 5)$ & $2(0,9 \pm 1)$ \\
\hline $\begin{array}{l}\text { 23. Calidad de la planificación, gestión } \\
\text { y administración }\end{array}$ & $73(33 \pm 3)$ & $75(34 \pm 6)$ & $65(29 \pm 6)$ & $10(5 \pm 3)$ & \\
\hline 25. ¿Está satisfecho con la formación pregrado? & $29(13 \pm 4)$ & $67(30 \pm 6)$ & $104(47 \pm 7)$ & $22(10 \pm 4)$ & $1(0,4 \pm 1)$ \\
\hline \multicolumn{6}{|l|}{ 7. Valore los componentes de la formación pregrado } \\
\hline Conocimientos teóricos & $5(2 \pm 2)$ & $15(7 \pm 3)$ & $85(38 \pm 6)$ & $84(37 \pm 6)$ & $36(16 \pm 5)$ \\
\hline Habilidades clínicas y técnicas & $84(37 \pm 6)$ & $77(34 \pm 6)$ & $46(20 \pm 5)$ & $17(8 \pm 4)$ & $1(0,4 \pm 1)$ \\
\hline Habilidades sociales y de comunicación & $41(18 \pm 5)$ & $46(20 \pm 5)$ & $68(30 \pm 6)$ & $56(25 \pm 6)$ & $14(6 \pm 3)$ \\
\hline Actitudes profesionales & $41(18 \pm 5)$ & $53(24 \pm 6)$ & $69(31 \pm 6)$ & $46(21 \pm 5)$ & $14(6 \pm 3)$ \\
\hline Aspectos éticos & $17(8 \pm 4)$ & $30(13 \pm 4)$ & $62(28 \pm 6)$ & $72(32 \pm 6)$ & $43(19 \pm 5)$ \\
\hline Otros & $24(26 \pm 6)$ & $12(13 \pm 4)$ & $44(48 \pm 7)$ & $9(9 \pm 4)$ & $3(3 \pm 2)$ \\
\hline
\end{tabular}

La tabla VI compara dos ítems en los dos años. Los conocimientos teóricos se valoran con una puntuación de 3 -una puntuación neutra en una escala de 1 a 5 - por el 38\% de los alumnos; en 1999, este porcentaje era del $14 \%$. En cuanto a las habilidades sociales y de comunicación, un $31 \%$ responde de manera favorable en 2008-2010, mientras que en 1999 era de un 5\%. Los aspectos éticos son importantes para un $51 \%($ IC $95 \%=51 \pm 7$ ) de los encuestados, mientras que este porcentaje es inferior (20\%) en 1999. Respecto a la calidad de la formación pregrado preclínica, un 37\% de los alumnos contestó de manera desfavorable, mientras que en 1999, los que contestaron de manera negativa fueron un $23 \%$.

De los encuestados, 208 alumnos (IC 95\% = 95 \pm 3 ) no se consideran suficientemente formados como médicos y 214 (IC 95\% = $96 \pm 3$ ) no se consideran capacitados para ejercer la medicina. El motivo que aluden es una formación práctica deficiente (cualitativa y cuantitativamente).

\section{Discusión}

Con este tipo de estudio se pretende poner de ma- nifiesto cuál es el grado de satisfacción del alumnado sobre las enseñanzas recibidas cuando alcanza su licenciatura [10]. Esta clase de observaciones permite conocer sus opiniones, tanto del proceso como de los resultados, con el objetivo, entre otros, de observar las deficiencias formativas que en su opinión presenta la formación de la medicina y así facilitar la toma de decisiones relativas a las actividades formativas $[2,11]$.

Los autores somos conscientes de los inconvenientes de la enseñanza estructurada en planes de estudio, basados fundamentalmente en asignaturas y en especialidades médicas, así como de las dificultades de coordinación, integración y trabajo en equipo en el desarrollo de los planes de estudio [12].

La participación en esta encuesta (70\%) ha sido ligeramente superior a la que se hizo en 1999, en las mismas condiciones y con las mismas características (62\%). Los resultados en cuanto a si los alumnos se encuentran formados y capacitados para ejercer la medicina son ligeramente inferiores a lo observado en el año 1999 (10\%) [9]. Esta observación se puso de manifiesto en el XVI Congreso Nacional de la SEDEM [13], donde se destacó que es uno de los mayores problemas que sufre la formación médica 
Tabla III. Formación del pregrado en medicina.

\begin{tabular}{|c|c|c|c|c|c|}
\hline \multirow{3}{*}{ Ítems } & \multicolumn{5}{|c|}{ Valoración general } \\
\hline & 1 & 2 & 3 & 4 & 5 \\
\hline & $n(\% \pm$ IC $95 \%)$ & $n(\% \pm$ IC 95\%) & $n(\% \pm$ IC 95\%) & $n(\% \pm I C 95 \%)$ & $n(\% \pm$ IC 95\%) \\
\hline \multicolumn{6}{|l|}{ 24. ¿Qué aspectos mejoraría? } \\
\hline Métodos de enseñanza & $9(4 \pm 3)$ & $14(6 \pm 3)$ & $47(21 \pm 5)$ & $59(26 \pm 6)$ & $95(42 \pm 6)$ \\
\hline Relación alumno-profesor & $11(5 \pm 3)$ & $21(9 \pm 4)$ & $39(17 \pm 5)$ & $63(28 \pm 6)$ & $90(40 \pm 6)$ \\
\hline Recursos empleados en la enseñanza & $11(5 \pm 3)$ & $21(9 \pm 4)$ & $47(21 \pm 5)$ & $59(27 \pm 6)$ & $85(38 \pm 6)$ \\
\hline Sistema de evaluación & $12(5 \pm 3)$ & $22(10 \pm 4)$ & $59(27 \pm 6)$ & $58(26 \pm 6)$ & $70(32 \pm 6)$ \\
\hline Gestión, planificación y administración & $14(6 \pm 3)$ & $12(5 \pm 3)$ & $27(12 \pm 4)$ & $48(22 \pm 5)$ & $122(55 \pm 7)$ \\
\hline Otros & $59(63 \pm 6)$ & $14(15 \pm 5)$ & $16(17 \pm 5)$ & $3(3 \pm 2)$ & $1(1,1 \pm 1)$ \\
\hline \multicolumn{6}{|l|}{$\begin{array}{l}\text { 19. ¿Qué fuente de conocimiento ha utilizado } \\
\text { como apoyo en su formación pregrado? }\end{array}$} \\
\hline Libros & $11(5 \pm 3)$ & $30(14 \pm 5)$ & $74(34 \pm 6)$ & $62(29 \pm 6)$ & $39(18 \pm 5)$ \\
\hline Revistas & $138(79 \pm 5)$ & $28(16 \pm 5)$ & $5(3 \pm 2)$ & $2(1 \pm 1)$ & $2(1 \pm 1)$ \\
\hline Apuntes elaborados & $26(13 \pm 4)$ & $21(11 \pm 4)$ & $34(17 \pm 5)$ & $40(20 \pm 5)$ & $78(39 \pm 6)$ \\
\hline Apuntes clase & $3(1 \pm 1)$ & $4(2 \pm 2)$ & $8(4 \pm 3)$ & $25(11 \pm 4)$ & $181(82 \pm 5)$ \\
\hline Bases de datos & $136(79 \pm 5)$ & $27(16 \pm 5)$ & $5(3 \pm 2)$ & $3(2 \pm 2)$ & $1(0,6 \pm 1)$ \\
\hline Internet & $83(45 \pm 7)$ & $40(22 \pm 5)$ & $37(20 \pm 5)$ & $21(11 \pm 4)$ & $3(2 \pm 2)$ \\
\hline Otros & $59(63 \pm 6)$ & $14(15 \pm 5)$ & $16(17 \pm 5)$ & $3(3 \pm 2)$ & $1(1 \pm 1)$ \\
\hline
\end{tabular}

en España. Dado que es grado, no prepara para el ejercicio, puesto que para ejercer en España, en el sistema sanitario español, es necesario obtener un título de especialidad vía MIR. Por ello, resulta preciso que la universidad mejore las competencias y habilidades que se dan a los alumnos, para que éstos puedan aprovechar al máximo su formación posterior. Este resultado concuerda con lo observado en otros apartados, como la escasa formación práctica y de habilidades clínicas. Sólo un 10\% (IC $95 \%=10 \pm 4$ ) se muestra satisfecho en cuanto a la calidad de su formación, resultado inferior al observado en el estudio de 1999 [9]. Se trata de distintos planes de estudios, lo cual puede suponer que este nuevo plan [7] no cubre las deficiencias del anterior [5]. De ahí que sea oportuna la puesta en marcha de los nuevos proyectos de grado, enmarcados en el proceso de convergencia europeo (EEES). Este proceso ha exigido, entre otras medidas, racionalizar los contenidos teóricos del grado, por la cuota de presencialidad en las clases teóricas, y cambiar los roles de los discentes y docentes, así como una planificación docente que garantice la continuidad del grado con el doctorado, por ser etapas formativas de un mismo proceso [14], entre la función básica universitaria, el posgrado o especialidad y el desarrollo profesional continuo.

Sólo un 10,4\% (IC 95\% = 10,4 \pm 4 ) de los encuestados se muestra satisfecho en cuanto a su formación; el 53\% (IC 95\% = $53 \pm 7$ ) está conforme con los conocimientos teóricos, resultado bastante inferior a lo observado en el estudio de 1999 [9]. En cuanto a las habilidades clínicas y técnicas, un $71 \%$ (IC 95\% = $71 \pm 6$ ) se muestra insatisfecho. Estos resultados/opiniones ponen de manifiesto que es necesario reducir el número de clases magistrales y dejar más tiempo para las prácticas, seminarios y otros procesos de autoaprendizaje -aspectos potenciados en el EEES [15]-, así como introducir a los estudiantes en el mundo sanitario, con el objetivo de que entiendan el rol y el impacto social y sanitario de su profesión [16]. El proyecto docente, puesto en marcha en este curso 2010-2011, se centra en el alumno, formando en los aspectos científicos y téc- 
Tabla IV. Formación del pregrado y MIR: respuestas afirmativas.

\begin{tabular}{|c|c|}
\hline Ítems & $n(\% \pm I C 95 \%)$ \\
\hline 10. ¿Le parece que el pregrado está condicionado por el examen MIR & $69(75 \pm 6)$ \\
\hline 11. ¿Le parece que los contenidos del pregrado están condicionados? & $154(70 \pm 6)$ \\
\hline 12. ¿Le parece que los métodos de enseñanza del pregrado están condicionados? & $130(58 \pm 6)$ \\
\hline 13. ¿Su aprendizaje ha estado condicionado? & $120(54 \pm 7)$ \\
\hline 14. ¿Es la prueba MIR un adecuado sistema de selección para la formación especializada? & $108(48 \pm 7)$ \\
\hline 15. ¿Le parece que deberían introducirse cambios en esta prueba? & $185(84 \pm 5)$ \\
\hline 16. Colaboración y coordinación entre áreas de conocimiento & $21(9 \pm 4)$ \\
\hline 17. ¿Le parecen adecuados los sistemas de evaluación? & $115(51 \pm 7)$ \\
\hline 18. ¿Le parecen adecuados los materiales y equipos? & $64(28 \pm 6)$ \\
\hline 26. ¿Conoce los cambios en relación a la adaptación al EEES? & $34(15,5 \pm 5)$ \\
\hline
\end{tabular}

Tabla V. Comparación de la formación del pregrado y MIR, estudios de 1999 y 2008-2010: respuestas afirmativas.

\begin{tabular}{|c|c|c|}
\hline \multirow{2}{*}{ Ítems } & \multicolumn{2}{|c|}{$n(\% \pm I C 95 \%)$} \\
\hline & $2008-2010$ & 1999 \\
\hline 10. ¿Le parece que el pregrado está condicionado por el examen MIR? & $169(75 \pm 6)$ & $100(87 \pm 4)$ \\
\hline 11. ¿Le parece que los contenidos del pregrado están condicionados? & $154(70 \pm 6)$ & $84(73 \pm 6)$ \\
\hline $\begin{array}{l}\text { 12. ¿Le parece que los métodos de enseñanza del pregrado } \\
\text { están condicionados? }\end{array}$ & $130(58 \pm 6)$ & $81(71 \pm 6)$ \\
\hline 13. ¿Su aprendizaje ha estado condicionado? & $120(54 \pm 7)$ & $52(45 \pm 7)$ \\
\hline $\begin{array}{l}\text { 14. ¿Es la prueba MIR un adecuado sistema de selección } \\
\text { para la formación especializada? }\end{array}$ & $108(48 \pm 7)$ & $28(24 \pm 6)$ \\
\hline 15. ¿Le parece que deberían introducirse cambios en esta prueba? & $185(84 \pm 5)$ & $101(88 \pm 4)$ \\
\hline
\end{tabular}

nicos y también en la capacidad de adquirir competencias, conocimientos y actitudes para la toma de decisiones en relación con el enfermo, implicándose en su formación activa desde el punto de vista humano, ético y social [14].

El 93\% (IC 95\% = $93 \pm 3$ ) de los alumnos refieren que utilizan como fuente de conocimiento los apuntes de clase, porcentaje bastante superior al $61 \%$ encontrado en el estudio del año 1999 [9]. Este hecho muestra que, cada año, los alumnos denotan mayor interés por asistir a clase, pero que también son po- cos los estudiantes que manejan otras fuentes, probablemente debido a la excesiva información que dan los profesores y al menor tiempo que dedican a la preparación de los exámenes. Es de esperar que el EEES sirva para cambiar esta dinámica y mejorar el proceso. Los libros son consultados por un $47 \%$ (IC 95\% = $47 \pm 7$ ), lo cual indica una escasa utilización de la biblioteca y, por tanto, refleja que el estudiante demuestra poco interés por ampliar y contrastar sus conocimientos. A pesar de las nuevas tecnologías de Internet y accesos similares a las bases de datos como Medline, EMBASE, Cochrane y otros, no se utilizan como herramienta habitual en el proceso de aprendizaje.

Los resultados, como se puede observar, son en parte desalentadores: aunque en la encuesta se refleja que adquieren conocimientos teóricos, la realidad es que no se sienten preparados para afrontar situaciones clínicas diarias, las cuales requieren una buena base científica y clínica. Por tanto, es importante considerar que los planes de grado deben desarrollar mayor número de competencias, habilidades y estrategias, como la introducción de pruebas de evaluación de competencias -evaluación clínica objetiva estructurada (ECOE) [17]-, para dar una formación integral (científica y práctica) que ofrezca mayor autoestima, seguridad y satisfacción [12].

Una gran mayoría opina que hay que mejorar los aspectos relacionados con los métodos de enseñanza (68\%), la relación alumno-profesor (68\%), el sistema de evaluación (58\%) y, sobre todo, la gestión, planificación y administración (77\%) de la formación, lo cual implica que parte de esas carencias pueden ser consecuencia del personal docente y de la gestión del proceso en su conjunto. También conviene reflexionar [18] sobre el sistema de evaluación porque los alumnos refieren que los exámenes tipo test no son una herramienta adecuada para valorar la competencia clínica y las habilidades en la resolución de problemas, ya que sólo potencia la memoria de datos e información, en ocasiones con poca o escasa relación clinicopatológica.

Se opina que el MIR condiciona el grado -el proceso y evaluación-, aunque en menor medida que lo referido en el año 1999 (75\% frente a 87\%) [9]. Para una gran mayoría, esta prueba condiciona desde los primeros cursos, tanto los contenidos como los métodos y el aprendizaje. También, al igual que en 1999 [9], ponen de manifiesto la necesidad de introducir cambios en la prueba MIR (84\%); sobre todo ahora, debido a que no se cubren todas las plazas, deben priorizarse otros objetivos frente al de selección de personal sanitario, como 
servir mejor para cumplir las expectativas profesionales que tienen nuestros graduandos (médicos). En este sentido, algunos autores [13] piensan que, puesto que los estudiantes de sexto curso pasan más tiempo en las academias preparando el examen MIR que en la facultad, es necesario plantearse la posibilidad de preparar a los estudiantes para la prueba MIR. No somos de esta opinión, y menos cuando se está empezando a implantar el grado. Éste debe cumplir el objetivo de dar formación básica, integral y estructurada, sobre los fundamentos de la medicina. Recientemente, con la reducción de porcentaje de valoración (del $25 \%$ al $10 \%$ ) del expediente, por una orden ministerial que alterará el proceso de enseñanza de las facultades de medicina, se da una mayor importancia a la formación en academias (examen: 90\%).

Debemos ser conscientes del importante momento que estamos viviendo en las facultades de medicina, que se manifiesta a través de múltiples eventos, seminarios, conferencias o artículos sobre educación médica, donde se constata una preocupación por cubrir las lagunas formativas y contribuir a mejorar la calidad de los estudios del grado.

Hay que preparar de la mejor manera posible a quienes van a ejercer la medicina del siglo XXI, cuando los objetivos propuestos para una buena formación médica de grado (según la Federación Mundial de Educación Médica para la formación de médicos de la Unión Europea) serían: preparar médicos eficientes en el campo asistencial, preventivo y sanitario; adecuar los programas educativos a las necesidades; integrar los conocimientos teóricos y la formación práctica, y establecer mecanismos de interconexión y continuidad entre los objetivos y programas educativos de la formación pregraduada, formación posgraduada y formación continua $[12,19,20]$.

En el estudio [9] de 1999 se dejaba una puerta abierta a la mejora de la situación con los nuevos planes de estudio. Lamentablemente, la situación es muy semejante y no ha mejorado, ya que los alumnos siguen manifestando que no están capacitados para ejercer la medicina. Las facultades de medicina no pueden convertirse en una academia de preparación del examen MIR, sino, por el contrario, tener claro su papel prioritario de convertir bachilleres en médicos aplicando, con distintas estrategias y procedimientos docentes, los fundamentos de la medicina $[21,22]$.

Debemos ser conscientes de la insatisfacción de los estudiantes y reevaluar el proyecto docente de cada asignatura y de cada facultad de medicina en su conjunto. El EEES supone, aunque sin medios, una oportunidad para mejorar y para que tanto dis-
Tabla VI. Comparación de distintos ítems.

\begin{tabular}{|c|c|c|c|c|c|}
\hline & \multicolumn{5}{|c|}{ Valoración } \\
\hline & 1 & 2 & 3 & 4 & 5 \\
\hline \multicolumn{6}{|c|}{ 7. Valore los componentes del pregrado } \\
\hline \multicolumn{6}{|c|}{ Conocimientos teóricos } \\
\hline $2008-2010$ & $2 \%$ & $7 \%$ & $38 \%$ & $37 \%$ & $16 \%$ \\
\hline 1999 & $2 \%$ & $4 \%$ & $14 \%$ & $37 \%$ & $43 \%$ \\
\hline \multicolumn{6}{|c|}{ Habilidades sociales y de comunicación } \\
\hline $2008-2010$ & $18 \%$ & $20 \%$ & $30 \%$ & $25 \%$ & $6 \%$ \\
\hline 1999 & $37 \%$ & $37 \%$ & $20 \%$ & $4 \%$ & $1 \%$ \\
\hline \multicolumn{6}{|l|}{ Aspectos éticos } \\
\hline $2008-2010$ & $8 \%$ & $13 \%$ & $28 \%$ & $32 \%$ & $19 \%$ \\
\hline 1999 & $25 \%$ & $23 \%$ & $32 \%$ & $14 \%$ & $6 \%$ \\
\hline \multicolumn{6}{|c|}{ 21. Calidad de la formación pregrado preclínica } \\
\hline $2008-2010$ & $9 \%$ & $28 \%$ & $35 \%$ & $25 \%$ & $3 \%$ \\
\hline 1999 & $6 \%$ & $17 \%$ & $50 \%$ & $26 \%$ & $0 \%$ \\
\hline
\end{tabular}

centes como docentes obtengan una mayor satisfacción y efectividad en la formación de la medicina, con unos mejores resultados.

Bibliografía

1. O'Neil EH. Education as part of the health care solution. JAMA 1992; 268: 1146-8.

2. Rozman C. La educación médica en el umbral del siglo XxI. Med Clin (Barc) 1997; 108: 582-6.

3. Penzo W. Enseñanza teórica y enseñanza práctica: ¿una clasificación pertinente? Med Clin (Barc) 1995; 105: 654-7.

4. Rodríguez-Artalejo F, Banegas-Banegas JR, RodríguezArtalejo A, Rodríguez-Artalejo C. Un modelo para el análisis de la actividad académica en medicina. Med Clin (Barc) 1997; 108: 499-502.

5. Universidad de Salamanca. Resolución de 15 de diciembre sobre el plan de estudios de Licenciado en Medicina. Madrid: BOE de 19 de enero de 1996; 17: 131-9.

6. Álvarez-Dardet C, Bolumar F, Porta M. Tipos de estudio. Med Clin (Barc) 1987; 89: 296-301.

7. Universidad de Salamanca. Facultad de Medicina. Guía académica 2008-2009. Salamanca: Gráficas Varona; 2008.

8. Argimón-Pallás JM, Jiménez-Villa J. Métodos de investigación clínica y epidemiológica. Barcelona: Elsevier; 2006.

9. Mirón-Canelo JA, Sáenz-González MC. Valoraciones y opiniones de los estudiantes de medicina sobre la formación del pregrado. Educ Med 1999; 2: 53-8.

10. Baum F. Investigación en salud pública: el debate sobre metodologías cuantitativas y cualitativas. Revisiones de Salud Pública 1997; 5: 175-93.

11. Escanero JF. La reforma del plan de estudios en las facultades de medicina. Rev Clin Esp 1992; 190: 274-7.

12. Pedraza-Muriel V. Formación médica y necesidades sociales. Med Clin (Barc) 2001; 116: 658-63. 
13. Moreno P. Hay que mejorar la coordinación entre la formación pregrado y MIR. URL: http://www.diariomedico.com.

14. Caramés J. Retos de la formación pregrado. URL: http:// www.diariomedico.com.

15. Bonete-Perales R. La adaptación de la Universidad de Salamanca al Espacio Europeo de Educación Superior: un desafío para todos. Salamanca: Univ. de Salamanca; 2006.

16. Baños JE. Algunas reflexiones sobre formación médica y necesidades sociales. Med Clin (Barc) 2002; 118: 636.

17. Gómez JM, Borrel F, Pujol R, Carretero JM, Pi F, Vilar F. Análisis del impacto del proyecto COMBELL sobre la competencia clínica médica. An Med Intern 1997; 14: 534-7.
18. Godfrey RC. Undergraduate examinations - a continuing tyranny. Lancet 1995; 345: 765-77.

19. World Federation for Medical Education. World Conference on Medical Education Report. Edinburgh, agosto de 1988.

20. Advisory Committee on Medical Training. Report and Recommendations on Undergraduate. Medical Education. Brussels, octubre de 1992.

21. Palés-Argullós J. ¿Una Bolonia en cada facultad de medicina o una sola Bolonia? Educ Med 2009; 12 (Supl 3): S7-11.

22. Gutiérrez-Fuentes JA. La implicación de todo el profesorado: un elemento clave. Estudio 'Enseñar a ser médico'. Educ Med 2009; 12 (Supl 3): S15-20. 